\title{
Dispersion sum rules for pion polarizabilities
}

\author{
Lev Fil'kov* \\ Lebedev Physical Institute \\ E-mail: filkovesci.lebedev.ru \\ Viktor Kashevarov \\ Lebedev Physical Institute \\ E-mail: kashev@kph.uni-mainz.de
}

An analysis of different dispersion sum rules (DSRs) for the dipole and quadrupole pion polarizabilities is carried out. We prove the absence of additional spurious singularities in these approaches. The results of the calculations of the polarizabilities in the framework of DSRs are compared with ChPT predictions and with data obtained in different experiments.

6th International Workshop on Chiral Dynamics

July 6-10, 2009

Bern, Switzerland

${ }^{*}$ Speaker. 


\section{Introduction}

Pion polarizabilities are fundamental structure parameters characterizing the behavior of the pion in an external electromagnetic field. The dipole electric $\left(\bar{\alpha}_{1}\right)$ and magnetic $\left(\bar{\beta}_{1}\right)$ pion polarizabilities measure the response of the pion to quasistatic electric and magnetic fields. On the other hand, the quadrupole polarizabilities $\bar{\alpha}_{2}$ and $\bar{\beta}_{2}$ measure the electric and magnetic quadrupole moments induced in the pion in the presence of an applied field gradient.

The generalized dipole $\left(\alpha_{1}\right.$ and $\left.\beta_{1}\right)$ and quadrupole $\left(\alpha_{2}\right.$ and $\left.\beta_{2}\right)$ polarizabilities are defined $[1,2]$ through expansion of the non-Born helicity amplitudes of Compton scattering on the pion in powers of $t$ at fixed $s=\mu^{2}$

$$
\begin{aligned}
& M_{++}\left(s=\mu^{2}, t\right)=\pi \mu\left[2\left(\alpha_{1}-\beta_{1}\right)+\frac{t}{6}\left(\alpha_{2}-\beta_{2}\right)\right]+\mathscr{O}\left(t^{2}\right), \\
& M_{+-}\left(s=\mu^{2}, t\right)=\frac{\pi}{\mu}\left[2\left(\alpha_{1}+\beta_{1}\right)+\frac{t}{6}\left(\alpha_{2}+\beta_{2}\right)\right]+\mathscr{O}\left(t^{2}\right),
\end{aligned}
$$

where $s=\left(q_{1}+k_{1}\right)^{2}, t=\left(k_{1}-k_{2}\right)^{2}\left(q_{1}, q_{2}\right.$ and $k_{1}, k_{2}$ are the pion and photon four-momenta), and $\mu$ is the pion mass. In the following the dipole and quadrupole polarizabilities are given in units $10^{-4} \mathrm{fm}^{3}$ and $10^{-4} \mathrm{fm}^{5}$, respectively.

It should be noted that the generalized pion polarizabilities very strongly differ from intrinsic polarizabilities $\bar{\alpha}_{i}$ and $\bar{\beta}_{i}$ [3]. For example, the nonrelativistic expression of the generalized electric dipole polarizability $\alpha_{1}$ is equal to

$$
\alpha_{1}=\bar{\alpha}_{1}+\frac{\alpha}{3 \mu}<r_{\pi}^{2}>=\bar{\alpha}_{1}+15.5
$$

where $\left\langle r_{\pi}^{2}>\right.$ is the mean-square pion radius, $\alpha$ is the fine-structure constant.

The expression for the generalized magnetic dipole polarizability $\beta_{1}$ is more complicated. Additional contributions could be both paramagnetic and diamagnetic. So, it is difficult to determine the nature of magnetic susceptibility of $\beta_{1}$ at present.

In the following we will omit the word "generalized".

The values of the pion polarizabilities are very sensitive to predictions of different theoretical models. Therefore, accurate experimental determination of these parameters is very important for testing the validity of such models.

For example, the results of calculations of $\left(\alpha_{1}-\beta_{1}\right)_{\pi^{ \pm}}$in Refs [2,4-6] are at variance with the predictions of chiral perturbation theory (ChPT) [7,8]. On the other hand, P. Pasquini, D. Drechsel, and S. Scherer (PDS) in Ref. [9] claim that as the absorptive part of the Compton amplitudes in Refs [2,4-6] is expressed by Breit-Wigner poles with energy dependent coupling constants and decay widths, there must appear additional spurious singularities. As a result, the values of the polarizabilities obtained in $[2,4-6]$ have to be modified essentially.

In the present paper we examine the statement of PDS and give an overview of the present situation in the field of investigation of the dipole and quadrupole polarizabilities of the charged and neutral pions in the frameworks of different DSRs and ChPT and compare the results of calculations with available experimental data. 


\section{Dispersion sum rules for dipole polarizabilities}

DSRs for the difference and sum of electric and magnetic pion polarizabilities have been constructed using dispersion relations (DRs) for the helicity amplitudes $M_{++}$and $M_{+-}$, respectively. It has been shown in Ref. [10] that these amplitudes have no kinematical singularities or zeroes.

In Refs $[2,4,6]$ DSR has been constructed for $\left(\alpha_{1}-\beta_{1}\right)$ using DR for the amplitude $M_{++}$at fixed $u=\mu^{2}$ (where $u=2 \mu^{2}-s-t$ ). In this case, the Regge-pole model allows the use of DR without subtractions [10]. Such a DSR is

$$
\left(\alpha_{1}-\beta_{1}\right)=\frac{1}{2 \pi^{2} \mu}\left(\int_{4 \mu^{2}}^{\infty} \frac{\operatorname{Im} M_{++}\left(t^{\prime}, u=\mu^{2}\right) d t^{\prime}}{t^{\prime}}+\int_{4 \mu^{2}}^{\infty} \frac{\operatorname{Im} M_{++}\left(s^{\prime}, u=\mu^{2}\right) d s^{\prime}}{s^{\prime}-\mu^{2}}\right) .
$$

The imaginary parts of the amplitudes in these DR and DSR are approximated by the contributions of meson resonances using Breit-Wigner expressions. For example, the contributions of the vector mesons are determined as

$$
\operatorname{Im} M_{++}^{(V)}(s, t)=-4 g_{V}^{2} s \frac{\Gamma_{0}}{\left(M_{V}^{2}-s\right)^{2}+\Gamma_{0}^{2}}
$$

for $s>4 \mu^{2}$ and $\operatorname{Im} M_{++}^{(V)}(s, t)=0$ for $s<4 \mu^{2}$, where

$$
g_{V}^{2}=6 \pi \sqrt{\frac{M_{V}^{2}}{s}}\left(\frac{M_{V}}{M_{V}^{2}-\mu^{2}}\right)^{3} \Gamma_{V \rightarrow \gamma \pi}, \quad \Gamma_{0}=\left(\frac{s-4 \mu^{2}}{M_{V}^{2}-4 \mu^{2}}\right)^{\frac{3}{2}} M_{V} \Gamma_{V} .
$$

Here $M_{V}, \Gamma_{V}$, and $\Gamma_{V \rightarrow \gamma \pi}$ are the mass, full and $\gamma \pi$ decay widths of the vector mesons, respectively. A dependence of the width on the energy is conditioned by the threshold behavior. The energy dependence of the coupling constant $g_{V}$ appears via an expression for the total cross section of the process $\gamma \pi \rightarrow \gamma \pi$ through the vector meson contribution.

In order to check the possibility of the appearance of additional singularities in our dispersion approach, we calculate the contributions of all mesons, except $\sigma$, to our DSR by the zero-width approximation.

The results of such calculations of $\left(\alpha_{1}-\beta_{1}\right)_{z}$ are listed in Tables 1, 2 together with the complete calculations of $\left(\alpha_{1}-\beta_{1}\right)_{f}$ obtained in Ref. [2].

Table 1: The DSR predictions for $\left(\alpha_{1}-\beta_{1}\right)_{\pi^{ \pm}}$.

\begin{tabular}{cccccccccc}
\hline & $\rho$ & $b_{1}$ & $a_{1}$ & $a_{2}$ & $f_{0}$ & $f_{0}^{\prime}$ & $\sigma$ & $\Sigma$ & $\Delta \Sigma$ \\
\hline$\left(\alpha_{1}-\beta_{1}\right)_{f}$ & -1.15 & 0.93 & 2.26 & 1.51 & 0.58 & 0.02 & 9.45 & 13.60 & 2.15 \\
\hline$\left(\alpha_{1}-\beta_{1}\right)_{z}$ & -1.11 & 0.85 & 3.39 & 1.51 & 0.59 & 0.03 & 9.45 & 13.70 & \\
\hline
\end{tabular}

As seen from the Tables the zero-width approximation results practically coincide with the calculations of Ref. [2] which are beyond such an approximation.

The coefficient $1 / \sqrt{t}$ in the coupling constant of the $\sigma$-meson amplitude in Ref. [2,4] provides the correct asymptotic behavior for the convergence of the integral over $t$ in our DSR and does not lead to additional singularities. To check it we have calculated this integral using the energy 
Table 2: The DSR predictions for $\left(\alpha_{1}-\beta_{1}\right)_{\pi^{0}}$.

\begin{tabular}{ccccccccc}
\hline & $\rho$ & $\omega$ & $\phi$ & $f_{0}$ & $f_{0}^{\prime}$ & $\sigma$ & $\Sigma$ & $\Delta \Sigma$ \\
\hline$\left(\alpha_{1}-\beta_{1}\right)_{f}$ & -1.58 & -12.56 & -0.04 & 0.60 & 0.02 & 10.07 & -3.49 & 2.13 \\
\hline$\left(\alpha_{1}-\beta_{1}\right)_{z}$ & -1.99 & -11.81 & -0.04 & 0.61 & 0.02 & 10.07 & -3.14 & \\
\hline
\end{tabular}

independent values of the decay width and the coupling constant of the $\sigma$-meson. It has not lead to essential changes of the calculation results presented in the Tables. For example, the value of $\left(\alpha_{1}-\beta_{1}\right)_{\pi^{ \pm} z}$ would be equal to 13.1 .

Besides, we compare our DSR calculation results in the $s$-channel with the predictions of DSR obtained at the fixed angle $\theta_{\gamma \pi}=180^{\circ}$ [5]. In this case

$$
\left(\alpha_{1}-\beta_{1}\right)^{(s)}=\frac{1}{2 \pi^{2}} \int_{3 \mu / 2}^{\infty} \frac{d v}{v^{2}}\left[1+\frac{v}{\mu}\right][\sigma(\text { yes })-\sigma(\text { no })],
$$

where $v$ is the incident photon energy in the lab. system, $\sigma(y e s)$ and $\sigma(n o)$ stand for the sum of the photoabsorption cross sections containing, respectively, parity-flip and -nonflip multipoles.

The best way to calculate this integral would be the use of experimental values for the cross sections. On the other hand, if these experimental data are well described by some function in the physical region of the process under consideration, then we can use this function, but only in this region, without continuation into unphysical regions. Usual Breit-Wigner forms for the photoabsorption cross sections with energy dependent decay widths and correct asymptotic behavior are such functions. Therefore, we should use them only in the physical regions of these processes. If we add a contribution from spurious singularities, which are out of the physical region considered in DSR, we would have an additional contribution to the result obtained from the integration of the experimental cross section. This is a gross mistake. So, there are no problems with additional spurious singularities in the derivation and the calculation of Eq. (2.4). The results of the calculation of this expression are very close to the values of $\left(\alpha_{1}-\beta_{1}\right)_{f}$ given in Tables (1) and (2) for $\rho$, $\omega, \phi, a_{1}$, and $a_{2}$ mesons which saturate the DSR integrals in the $s$-channel. This result confirms the absence of additional singularities in our approach for the $s$-channel integral of our DSRs. The $t$-channel contributions with $I=J=0$ are the same for both DSR at fixed $u=\mu^{2}$ and DSR at fixed $\theta_{\gamma \pi}=180^{\circ}$. There are the same arguments why additional singularities are absent in the $t$-channel too.

It should be noted that in the work of S.S. Kamalov, L. Tiator, D. Drechsel et al. [11] the neutral pion photoproduction and electroproduction at the threshold were analyzed using DR. In this work the resonance contribution to the imaginary parts of the amplitudes of the pion photoproduction and electroproduction were given in terms of Breit-Wigner expressions with the energy dependent decay widths and coupling constants. According to the main statement of PDS, there are very many additional singularities in the resonance amplitudes considered in this work. The results of the calculations in the work [11] were obtained without consideration of any additional singularities and are in very good agreement with the experimental data for the pion photoproduction in the threshold region. However, if, according to PDS, one takes into account these singularities, this would lead to additional contributions and, as a result, to a disagreement with the experiment. This confirms that an account of such singularities is a mistake. 
It is worth noting that the calculation of $\left(\alpha_{1}-\beta_{1}\right)_{\pi^{ \pm}}$in the framework of DSR at finite energy [12], which takes into account the $s$-channel and the Regge-pole asymptotic contribution only, yielded $\left(\alpha_{1}-\beta_{1}\right)_{\pi^{ \pm}}=10.3 \pm 1.3$. This value practically coincides with our result (see Table 1).

As for the sum $\left(\alpha_{1}+\beta_{1}\right)$, these values are calculated using Baldin's DSR

$$
\left(\alpha_{1}+\beta_{1}\right)=\frac{1}{2 \pi^{2}} \int_{\frac{3}{2} \mu}^{\infty} \frac{\sigma_{T}(v) d v}{v^{2}},
$$

where $\sigma_{T}$ is the total cross section of the $\gamma \pi$-interaction. These DSR results in

$$
\begin{aligned}
& \left(\alpha_{1}+\beta_{1}\right)_{\pi^{ \pm}}=0.166 \pm 0.024 \\
& \left(\alpha_{1}+\beta_{1}\right)_{\pi^{0}}=0.802 \pm 0.035
\end{aligned}
$$

On the other hand, two-loop ChPT calculations [7,13] give

$$
\begin{aligned}
& \left(\alpha_{1}-\beta_{1}\right)_{\pi^{ \pm}}=5.7 \pm 1.0 \\
& \left(\alpha_{1}+\beta_{1}\right)_{\pi^{ \pm}}=0.16 \\
& \left(\alpha_{1}-\beta_{1}\right)_{\pi^{0}}=-1.9 \pm 0.2 \\
& \left(\alpha_{1}+\beta_{1}\right)_{\pi^{0}}=1.1 \pm 0.3 .
\end{aligned}
$$

So, the results of the ChPT calculations for the sum and difference of the dipole polarizabilities of $\pi^{0}$ and the sum for the charged pions do not conflict within the errors with predictions of DSRs.

Let us consider possible reasons of the discrepancy between the predictions of DSRs and ChPT for $\left(\alpha_{1}-\beta_{1}\right)_{\pi^{ \pm}}$. The main contribution to the DSRs for $\left(\alpha_{1}-\beta_{1}\right)_{\pi^{ \pm}}$is given by the $\sigma$-meson. However, this meson is taken into account only partially in the present ChPT calculations.

Consider the methods of the calculation of the vector meson contribution in the frameworks of DSRs and ChPT. In the narrow width approximation we have from Eq. (2.2)

$$
\operatorname{Im} M_{++}^{(V)}(s, t)=-4 \pi g_{V}^{2} s \delta\left(s-M_{v}^{2}\right) .
$$

Then the DSR calculation gives

$$
\operatorname{ReM}_{++}\left(s=\mu^{2}, t=0\right)=\frac{-4 g_{V}^{2} M_{V}^{2}}{\left(M_{V}^{2}-\mu^{2}\right)}
$$

In the case of ChPT the authors of Ref. [7] used

$$
\operatorname{ReM}_{++}\left(s=\mu^{2}, t=0\right)=\frac{-4 g_{V}^{2} \mu^{2}}{\left(M_{V}^{2}-\mu^{2}\right)} .
$$

The absolute value of the amplitude (2.9) is smaller than (2.8) by a factor $M_{V}^{2} / \mu^{2}$. From the point of view of analyticity, the result (2.9) could be obtained if DR with one subtraction at $s=0$ is used for the amplitude $M_{++}(s, t)$. However, an additional subtraction constant $M_{++}(s=0, t=0)$ then appears, which was not considered in the available ChPT calculations.

In the case of the difference of the dipole polarizabilities of the $\pi^{0}$-meson, the big contribution of the $\sigma$-meson to DSR is cancelled by the big contribution of the $\omega$-meson. On the other hand, in the ChPT calculations the $\sigma$-meson is only partially included and the $\omega$-meson gives a very small contribution to this difference. As a result, the DSR and ChPT predictions for $\left(\alpha_{1}-\beta_{1}\right)_{\pi^{0}}$ are rather close. 
Table 3: The experimental data presently available for $\left(\alpha_{1}-\beta_{1}\right)_{\pi^{ \pm}}$. In [16-20] $\left(\alpha_{1}-\beta_{1}\right)_{\pi^{ \pm}}$was determined by using the constraint $\alpha_{1 \pi^{ \pm}}=-\beta_{1 \pi^{ \pm}}$.

\begin{tabular}{|c|c|}
\hline Experiments & $\left(\alpha_{1}-\beta_{1}\right)_{\pi^{ \pm}}$ \\
\hline MAMI (2005) [14] & $11.6 \pm 1.5_{\text {stat }} \pm 3.0_{\text {syst }} \pm 0.5_{\text {mod }}$ \\
\hline Lebedev Phys. Inst. (1984) [15] & $40 \pm 24$ \\
\hline$\pi^{-} A \rightarrow \gamma \pi^{-} A \quad$ Serpukhov (1983) [16] & $13.6 \pm 2.8 \pm 2.4$ \\
\hline$\pi^{-} A \rightarrow \gamma \pi^{-} A \quad$ COMPASS (2007) [17] & 5.0 $\pm 3.4 \quad$ (preliminary) \\
\hline \multicolumn{2}{|l|}{  } \\
\hline $\begin{array}{l}\text { D. Babusci et al. (1992) [18] } \\
\text { PLUTO [21] } \\
\text { DM } 1 \text { [22] } \\
\text { DM } 2 \text { [23] } \\
\text { MARK II [24] }\end{array}$ & $\begin{array}{l}38.2 \pm 9.6 \pm 11.4 \\
34.4 \pm 9.2 \\
52.6 \pm 14.8 \\
4.4 \pm 3.2\end{array}$ \\
\hline $\begin{array}{l}\text { J.F. Donoghue, B.R. Holstein (1993) [19] } \\
\text { MARK II [24] }\end{array}$ & 5.4 \\
\hline $\begin{array}{l}\text { A.E. Kaloshin, V.V. Serebryakov (1994) [20] } \\
\text { MARK II [24] }\end{array}$ & $5.25 \pm 0.95$ \\
\hline \begin{tabular}{ll}
\multicolumn{1}{c}{ L.V. Fil'kov, V.L. Kashevarov (2006) [4] } \\
$\gamma \gamma \rightarrow \pi^{+} \pi^{-}$ & fit of data [24-29] \\
& from threshold to $2.5 \mathrm{GeV}$
\end{tabular} & $\begin{array}{l}13.0_{-1.9}^{+2.6} \\
\end{array}$ \\
\hline
\end{tabular}

\section{Experimental data for dipole polarizabilities of charged pions}

By now the values of the pion polarizabilities were determined by analyzing the processes $\pi^{-} A \rightarrow \gamma \pi^{-} A, \gamma p \rightarrow \gamma \pi^{+} n$, and $\gamma \gamma \rightarrow \pi \pi$. The experimental information available so far for the difference of the dipole polarizabilities of charged pions is summarized in Table 3.

The values of the experimental cross sections of the process $\gamma \gamma \rightarrow \pi^{+} \pi^{-}$in the energy region $E_{\gamma}<700 \mathrm{MeV}$ are very ambiguous. As a result, the values of $\left(\alpha_{1}-\beta_{1}\right)_{\pi^{ \pm}}$, obtained from analyses of these data, lie in the interval 4.4-52.6. The analyses of the data of Mark II [24] only have given $\left(\alpha_{1}-\beta_{1}\right)_{\pi^{ \pm}}$close to the ChPT result.

The difference $\left(\alpha_{1}-\beta_{1}\right)_{\pi^{ \pm}}$found from the global fit to all available experimental data of the process $\gamma \gamma \rightarrow \pi^{+} \pi^{-}$in the energy region from the threshold to $2500 \mathrm{MeV}$ [2] agrees very well with the results [16] obtained from the scattering of high energy $\pi^{-}$mesons off the Coulomb field of heavy nuclei and from the radiative photoproduction of $\pi^{+}$from the proton at MAMI [14] and in Lebedev Physical Institute [15] (see Table 3) and with the DSR calculations. However, these values of $\left(\alpha_{1}-\beta_{1}\right)_{\pi^{ \pm}}$deviate essentially from the ChPT calculations [7, 8].

Results of polarizability extraction from the process $\pi^{-} A \rightarrow \gamma \pi^{-} A$ strongly depend on the momentum transfer $Q^{2}$. In this reaction the Coulomb amplitude dominates for $Q^{2} \lesssim 10^{-4}(\mathrm{GeV} / \mathrm{c})^{2}$. In the region of $Q^{2} \sim 10^{-3}(\mathrm{GeV} / \mathrm{c})^{2}$ Coulomb and nuclear contributions are of similar size. In this region the nuclear contribution, in particular an interference between the Coulomb and nuclear amplitudes, should be taken into account. In the work [16] the authors considered $Q^{2}<6 \times 10^{-4}$ $(\mathrm{Gev} / \mathrm{c})^{2}$, while the authors of Ref. [17] worked at $Q^{2}<7.5 \times 10^{-3}(\mathrm{Gev} / \mathrm{c})^{2}$. In this region of $Q^{2}$ the 
Table 4: The quadrupole polarizabilities of the neutral and charged pions.

\begin{tabular}{|l|l|l|c|c|}
\hline & & & \multicolumn{2}{|c|}{ ChPT [7,13] } \\
\cline { 4 - 5 } & \multicolumn{1}{|c|}{ fit [2,4] } & DSR [2] & to one-loop & to two-loops \\
\hline$\left(\alpha_{2}-\beta_{2}\right)_{\pi^{0}}$ & $39.7 \pm 0.02$ & $39.72 \pm 8.01$ & & $37.6 \pm 3.3$ \\
\hline$\left(\alpha_{2}+\beta_{2}\right)_{\pi^{0}}$ & $-0.181 \pm 0.004$ & $-0.171 \pm 0.067$ & & 0.04 \\
\hline$\left(\alpha_{2}-\beta_{2}\right)_{\pi^{ \pm}}$ & $25.0_{-0.3}^{+0.8}$ & $25.75 \pm 7.03$ & 11.9 & $16.2[21.6]$ \\
\hline$\left(\alpha_{2}+\beta_{2}\right)_{\pi^{ \pm}}$ & $0.133 \pm 0.015$ & $0.121 \pm 0.064$ & 0 & $-0.001[-0.001]$ \\
\hline
\end{tabular}

contribution of the interference between the Coulomb and nuclear amplitudes is very large [30,31]. However, it was not taken into account in the work [17]. This is the main reason of the difference between the Serpukhov and COMPASS results. Moreover, for the total energy, in the $\gamma \pi$ c.m.s., $W \gtrsim 450 \mathrm{MeV}$ and $\theta_{\gamma \gamma^{\prime}} \sim 180^{\circ}$, the $\sigma$-meson contribution should be taken into account [6].

\section{Pion quadrupole polarizabilities}

DSRs for the difference and the sum of the quadrupole polarizabilities have been obtained with the help of DRs at fixed $u=\mu^{2}$ with one subtraction for the amplitudes $M_{++}$and $M_{+-}$, respectively:

$$
\begin{gathered}
\left(\alpha_{2}-\beta_{2}\right)=\frac{6}{\pi^{2} \mu}\left\{\int_{4 \mu^{2}}^{\infty} \frac{\operatorname{Im} M_{++}\left(t^{\prime}, u=\mu^{2}\right) d t^{\prime}}{t^{\prime 2}}-\int_{4 \mu^{2}}^{\infty} \frac{\operatorname{Im} M_{++}\left(s^{\prime}, u=\mu^{2}\right) d s^{\prime}}{\left(s^{\prime}-\mu^{2}\right)^{2}}\right\}, \\
\left(\alpha_{2}+\beta_{2}\right)=\frac{6 \mu}{\pi^{2}}\left\{\int_{4 \mu^{2}}^{\infty} \frac{I m M_{+-}\left(t^{\prime}, u=\mu^{2}\right) d t^{\prime}}{t^{\prime 2}}-\int_{4 \mu^{2}}^{\infty} \frac{I m M_{+-}\left(s^{\prime}, u=\mu^{2}\right) d s^{\prime}}{\left(s^{\prime}-\mu^{2}\right)^{2}}\right\} .
\end{gathered}
$$

The corresponding values of the quadrupole polarizabilities have been found in Refs $[2,4]$ by fitting the experimental total cross sections of the processes $\gamma \gamma \rightarrow \pi^{0} \pi^{0}[32,33]$ and $\gamma \gamma \rightarrow$ $\pi^{+} \pi^{-}$[24-29] in the energy regions from the thresholds to 2.25 and $2.5 \mathrm{GeV}$, respectively. The fit functions were constructed by using DRs with subtractions for the amplitudes $M_{++}$and $M_{+-}$.

The values of the quadrupole polarizabilities found in Refs. [2,4] and the predictions of DSRs [2] and ChPT [7,13] are listed in Table 4. The numbers in brackets correspond to the order $p^{6}$ low energy constants from Ref. [34]. As seen from this Table, all values of the polarizabilities found in Ref. [2,4] are in good agreement with the DSR predictions [2].

The difference of the quadrupole polarizabilities $\left(\alpha_{2}-\beta_{2}\right)_{\pi^{ \pm}}$obtained in Refs. [2,4] disagrees with the present two-loop ChPT calculations [7,13]. One of the sources of this disagreement is the bad knowledge of the low energy constants. Moreover, it should be noted that in this case the two-loop contribution generates nearly $100 \%$ as compared to the one-loop result. The ChPT calculations of $\left(\alpha_{2}+\beta_{2}\right)$ give an opposite sign. However, calculations of $\left(\alpha_{2}+\beta_{2}\right)$ at order $p^{6}$ determine only the leading order term in ChPT. Therefore, contributions at $p^{8}$ could be essential, and considerably more work is required to put the chiral prediction on a firm basis in this case [7].

It is worth noting that calculations of the dipole and quadrupole pion polarizabilities in the frame of the Nambu-Jona-Lasino model [35] agree within errors with the DSR [2,4] predictions. 


\section{Summary}

We showed that there are no problems with additional spurious singularities in the DSRs and DRs considered. The difference between the predictions of the DSRs and ChPT for $\left(\alpha_{1}-\beta_{1}\right)_{\pi^{ \pm}}$ remains. This discrepancy is connected with a different account of the contribution of the $\sigma$ and vector mesons in the DSR and ChPT calculations. The disagreement between the DSR and ChPT predictions of the quadrupole polarizabilities is connected, in particular, with the bad knowledge of the low energy constants. Substantial corrections to the values of the quadrupole polarizabilities are expected from three-loop calculations.

The authors thank A.I. L'vov and V.A. Petrun'kin for useful discussions. This research was supported by the DFG-RFBR (Grant No. 09-02-91330).

\section{References}

[1] I. Guiasu and E.E. Radescu, Ann. Phys. (NY) 120, 145 (1979); ibid. 122, 436 (1979).

[2] L.V. Fil'kov and V.L. Kashevarov, Phys. Rev. C 72, 035211 (2005).

[3] V.A. Petrun'kin, Nucl. Phys. 55, 197 (1964); Sov. J. Part. Nucl. 12, 278 (1981).

[4] L.V. Fil'kov and V.L. Kashevarov, Phys. Rev. C 73, 035210 (2006).

[5] L.V. Fil'kov, I. Guiasu, and E.E. Radescu, Phys. Rev. D 26, 3146 (1982).

[6] L.V. Fil'kov and V.L. Kashevarov, Eur. Phys. J. A 5, 285 (1999).

[7] J. Gasser, M.A. Ivanov, and N.E. Sainio, Nucl. Phys. B745, 84 (2006).

[8] U. Bürgi, Nucl. Phys. B479, 392 (1996).

[9] B. Pasquini, D. Drechsel, and S. Scherer, Phys. Rev. C 77, 065211 (2008).

[10] H.D.I. Abarbanel and M.L. Goldberger, Phys. Rev. 165, 1594 (1968).

[11] S.S. Kamalov, L. Tiator, D. Drechsel etal., Phys. Rev. C 66, 065206 (2002).

[12] A.I. L'vov and V.A. Petrun'kin, Sov. Phys.-Lebedev Inst. Rep. 12, 39 (1985).

[13] J. Gasser, M.A. Ivanov, and M.E. Sainio, Nucl. Phys. B728, 31 (2005).

[14] J. Ahrens et al., Eur. Phys. J. A 23, 113 (2005).

[15] T.A. Aibergenov et al. Sov. Phys.-Lebedev Inst. Rep. 6, 32 (1984); Czech. J. Phys. B 36, 948 (1986).

[16] Yu.M. Antipov et al., Phys. Lett. B121, 445 (1983).

[17] M. Colantoni, Proceed. of 11 Intern. Confer. on Meson-Nucleon Physics and Structure of the Nucleon, Juelich, Germany, 2007.

[18] D. Babusci et al., Phys. Lett. B277, 158 (1992).

[19] J.F. Donoghue and B.R. Holstein, Phys. Rev. D 48, 137 (1993).

[20] A.E. Kaloshin and V.V. Serebryakov, Z. Phys. C 64, 689 (1994).

[21] PLUTO Collaboration (C. Berger et al.), Z. Phys. C 26199 (1984).

[22] DM1 Collaboration (A. Courau et al.), Nucl. Phys. B271, 1 (1986). 
[23] DM2 Collaboration (Z. Ajaltoni et al.), Phys. Lett. B194, 573 (1987).

[24] Mark II Collaboration (J. Boyer et al.),Phys. Rev. D 42, 1350 (1990).

[25] TPC/2 $\gamma$ Collaboration (H. Aihara et al.), Phys. Rev. Lett. 57, 404 (1986).

[26] CELLO Collaboration (H.J. Behrend et al.), Z. Phys. C 56, 381 (1992).

[27] VENUS Collaboration (Fumiaki Yabuki et al.), J. Phys. Soc. Jap. 64, 435 (1995).

[28] ALEPH Collaboration (A. Heister et al.), Phys. Lett. B569, 140 (2003).

[29] Belle Collaboration (H. Makazawa et al.), Phys. Lett. B615, 39 (2005).

[30] L.V. Fil'kov and V.L. Kashevarov, Proceed. of "NSTAR 2007", Bonn, Germany, 05-08 September (2007), p.179; arXiv:0802.0965 (nucl-th).

[31] Th. Walcher, Progr. Part. Nucl. Phys. 61, 106 (2008).

[32] H. Marsiske et al., Phys. Rev. D 41, 3324 (1990).

[33] J.K. Bienlein, Crystal Ball Contribution to the 9th Intern. Workshop on Photon-Photon Collisions, San Diego, California 22-26 March 1992, ISSN 0418-9833.

[34] J. Bijnens and J. Prades, Nucl. Phys. B490, 239 (1997).

[35] B. Hiller et al., arXiv:0908.0159 (hep-ph). 